\title{
Características posoperatorias de la faringoplastia combinada con amigdalectomía en comparación con la amigdalectomía en niños con síndrome obstructivo de apnea del sueño Postoperative characteristics of combined pharyngoplasty and tonsillectomy versus tonsillectomy in children with obstructive sleep apnea syndrome
}

\author{
Dra. Yan Shu ${ }^{a}$, Dr. Hong-Bing Yao ${ }^{a}$, Dr. Da-Zhi Yang ${ }^{a}$ y Dr. Bing Wang ${ }^{a}$
}

\section{RESUMEN}

Objetivo. Identificar las ventajas posoperatorias de la amigdalectomía en conjunto con la faringoplastia en comparación con la amigdalectomía sola en niños con apnea obstructiva del sueño.

Métodos. En un estudio prospectivo observacional, los pacientes que cumplían con los criterios del estudio fueron asignados aleatoriamente a dos grupos: el grupo de amigdalectomía y faringoplastia, y el grupo de solo amigdalectomía. En ambos grupos, también se practicó adenoidectomía a los pacientes con vegetaciones. Se compararon los procesos de cicatrización en la herida faríngea y las proporciones hemorrágicas. Además, se evaluó la función velofaríngea posoperatoria.

Resultados. La faringoplastia junto con la amigdalectomía se practicó en 328 niños con apnea obstructiva del sueño debido a hipertrofia amigdalina, y la amigdalectomía sola, en 275 niños. Estas cohortes no mostraban diferencias demográficas significativas. Por otro lado, se encontró que la pérdida de sangre fue significativamente menor en el grupo que recibió amigdalectomía y faringoplastia $(\mathrm{p}<0,01)$, y el proceso de cicatrización fue notablemente más breve. La función velofaríngea posoperatoria no resultó afectada.

Conclusiones. En comparación con la amigdalectomía, la amigdalectomía junto con la faringoplastia redujeron la duración del proceso de cicatrización y la pérdida de sangre;no obstante, las complicaciones posoperatorias no aumentaron. En nuestra opinión, la combinación de faringoplastia y amigdalectomía reúne un gran potencial en el tratamiento de los niños con apnea obstructiva del sueño.

Palabras clave: Faringoplastia, amigdalectomía, niño, apnea obstructiva del sueño.

http:/ / dx.doi.org/10.5546/ aap.2018.316 Texto completo en inglés: http:/ / dx.doi.org/10.5546/ aap.2018.eng.316
Cómo citar: Shu Y, Yao HB, Yang DZ, Wang B. Características posoperatorias de la faringoplastia combinada con amigdalectomía en comparación con la amigdalectomía en niños con síndrome obstructivo de apnea del sueño Arch Argent Pediatr 2018;116(5):316-321.

\section{INTRODUCCIÓN}

El síndrome de apnea obstructiva del sueño es un trastorno frecuente y con alta prevalencia en los pacientes pediátricos, y afecta al 2\%-3\% de los niños. ${ }^{1}$ La incidencia máxima se encontró en los niños de preescolar, dado que esa es la edad en que la hipertrofia amigdalina y las adenoides se observan con mayor frecuencia. ${ }^{2}$ Si no se la trata, la apnea obstructiva del sueño puede derivar en una morbilidad grave y afectar los sistemas cognitivo, neuroconductual y cardiovascular., En los niños, la apnea obstructiva del sueño se debe principalmente a la hipertrofia adenoamigdalina; ${ }^{5}$ por lo tanto, la amigdalectomía con o sin adenoidectomía es, en la actualidad, el tratamiento habitual para los niños con apnea obstructiva del sueño. ${ }^{6}$ El procedimiento habitual para la adenoamigdalectomía incluye la extracción de las amígdalas palatinas y las vegetaciones adenoides.

Aunque la amigdalectomía es uno de los procedimientos otorrinolaringológicos más utilizados, es posible que surjan algunas complicaciones, como hemorragia posoperatoria, dolor, disminución de la ingesta oral, compromiso respiratorio posobstructivo, 
insuficiencia velofaríngea y trauma psicológico. ${ }^{7,8}$ Con el objeto de reducir estas complicaciones, se han ido generando diversas técnicas, incluida la ablación de las amígdalas por radiofrecuencia, ${ }^{9}$ la amigdalectomía mediante electrodisección bipolar ${ }^{10}$ y la amigdalectomía con láser. ${ }^{11}$ Sin embargo, no hay pruebas contundentes que respalden el uso de la faringoplastia en niños con apnea obstructiva del sueño. El término "faringoplastia" hace referencia al cierre de la herida amigdalina mediante la sutura de los pilares anterior y posterior después de la amigdalectomía. Durante mucho tiempo, la necesidad y el riesgo de suturar la herida amigdalina tras la amigdalectomía han generado controversias.

El objetivo de este estudio fue comparar los resultados de la amigdalectomía en conjunto con faringoplastia con los resultados de la amigdalectomía sola en niños con apnea obstructiva del sueño.

\section{MATERIALES Y MÉTODOS Población}

La población de nuestro estudio estuvo formada por pacientes pediátricos evaluados y que tuvieran una amigdalectomía programada, con o sin adenoidectomía, para tratar su apnea obstructiva del sueño en el Hospital Pediátrico de la Universidad Médica de Chongqing entre septiembre de 2008 y junio de 2010. Excluimos a los pacientes que solo se someterían a una adenoidectomía y observamos la excisión exclusiva de las amígdalas o la amigdalectomía con adenoidectomía. Las indicaciones para todos los pacientes incluían la presencia de apnea obstructiva del sueño y / o hipertrofia adenoide. El diagnóstico de apnea obstructiva del sueño se fundamentó en los antecedentes proporcionados por el cuidador (ronquido, respiración por la boca, apneas referidas por los convivientes, enuresis, hipersomnolencia diurna o sueño inquieto), el examen físico y los resultados del análisis de datos polisomnográficos. Elegimos pacientes con un índice de apnea/hipopnea (IAH) $>5$, establecido por polisomnografía, y todos los grados de amígdalas (I-IV). ${ }^{12}$ Los criterios de exclusión fueron comorbilidades importantes o antecedentes importantes de amigdalitis recurrente/crónica, además de antecedentes de trastorno hemorrágico. También excluimos los casos de obstrucción nasal crónica y otras causas de obstrucción de las vías respiratorias. Este estudio fue aprobado por el Comité de
Ética local. Los padres/tutores legales de los niños participantes firmaron un consentimiento informado. Los niños se dividieron según una tabla de números aleatorios en dos grupos de tratamiento: el grupo que se sometió a faringoplastia y amigdalectomía, y el grupo que solo recibió amigdalectomía.

En el laboratorio de trastornos del sueño, se les realizó una PSG nocturna, que se analizó mediante técnicas estándar. El índice de apnea/hipopnea (IAH) obstructiva se definió como la cantidad de apneas e hipoapneas por hora de todo el período de sueño. El tamaño de las vegetaciones se determinó por medio de una radiografía lateral del cuello. El cociente adenoide/nasofaringe $(\mathrm{A} / \mathrm{N})$ se define como el cociente entre el grosor máximo de la adenoide y la distancia a lo largo de una línea que se extiende desde el borde posterior-superior del paladar duro hasta la sincondrosis esfenooccipital en la base del cráneo. ${ }^{13}$ En nuestro estudio, se sometió a adenoidectomía a los pacientes con un $\mathrm{A} / \mathrm{N}>0,65$.

Algunos de los datos recogidos fueron edad y sexo del paciente, diagnóstico preoperatorio, resultados de la polisomnografía (PSG), clasificación de la inteligibilidad del habla y la insuficiencia velofaríngea, y cualquier complicación posoperatoria.

\section{Técnicas quirúrgicas}

Los niños de ambos grupos recibieron anestesia general y se les colocó intubación endotraqueal. Durante la cirugía, estuvieron en la posición supina habitual. Se les abrió la boca con un abrebocas de Boyle-Davis, y el paladar blando se retrajo con dos catéteres pediátricos de $9 \mathrm{~mm}$.

En el grupo de faringoplastia y amigdalectomía, tras identificar el plano de disección pericapsular, se realizó, con el máximo cuidado, la disección pericapsular desde el polo superior hacia el inferior de las amígdalas. Luego, las amígdalas se disecaron hacia el polo inferior con un disector romo. Las amígalas se extirparon desde la fosa amigdalina con un lazo de alambre y luego, para lograr la hemostasia, la fosa se taponó con un hisopo empapado en solución salina normal. Por supuesto, cuando fue necesario, se utilizaron suturas absorbibles y pinzas bipolares para garantizar la hemostasia. Los pilares anterior y posterior, y la organización de la fosa se suturaron con una sutura de catgut crómico 4-0 simple, para asegurar la hemostasia y cerrar la fosa amigdalina. La sutura no se quitó después de la operación. 
En el grupo que solo recibió amigdalectomía, se realizaron la extirpación quirúrgica del propio tejido amigdalino y el procedimiento primario de hemostasia, lo que es similar al procedimiento descrito anteriormente. Luego, se procuró la hemostasia de la fosa amigdalina mediante pinza de coagulación bipolar. Una vez completa la hemostasia de ambas fosas amigdalinas, la adenoide se extirpó mediante cirugía endoscópica.

\section{Evaluación de la función velofaríngea \\ Evaluación del habla}

Con el consentimiento de los padres, cinco días después de la operación hicimos una evaluación del habla y una nasofaringoscopia flexible. Todos accedieron al examen. Las evaluaciones del habla se obtuvieron a partir de los análisis de rutina del habla llevados a cabo por patólogos del habla pediátricos, que utilizaron una escala desarrollada en esta institución antes de la aceptación de los parámetros del habla universales. Los resultados del análisis se clasificaron con una escala de cinco puntos (0-4), en la que 0 indica "normal" y 4 , "grave". Una puntuación más baja en esta escala indicaba una disfunción menos importante. ${ }^{14}$

\section{Nasofaringoscopia flexible}

El mismo otorrinolaringólogo pediátrico hizo endoscopias nasales a los niños de ambos grupos antes y después de la cirugía. La visualización del puerto velofaríngeo se realizó utilizando un nasofaringoscopio flexible de fibra óptica. Se observaron el movimiento palatino, el movimiento de la pared faríngea lateral y el cierre velofaríngeo. El tamaño del espacio velofaríngeo se midió durante el cierre máximo en la fonación. Los resultados se clasificaron según la escala de Golding-Kushner. ${ }^{15}$ Las categorías de tamaño del espacio se definieron como amplio (cierre velofaríngeo $<50 \%$ ), moderado (cierre velofaríngeo entre el $50 \%$ y el $80 \%$ ) y pequeño (cierre velofaríngeo $>80 \%$ ).

\section{Análisis estadístico}

El análisis estadístico se realizó utilizando el programa estadístico SPSS para Windows. Las variables demográficas de edad y sexo se compararon mediante la prueba de $\chi^{2}$. Las complicaciones pre y posoperatorias, como hipernasalidad y regurgitación nasofaríngea, se compararon usando la prueba exacta de Fisher. Un valor de $\mathrm{p}<0,05$ se consideró significativo en términos estadísticos.

\section{RESULTADOS \\ Demografía de las cohortes}

Los datos demográficos de los pacientes incluidos en este estudio se presentan en la Tabla 1. Con el fin de detectar apnea obstructiva del sueño, se evaluó un total de 682 pacientes de los cuales 79 se excluyeron porque sus padres se negaron a la endoscopia nasal posoperatoria. Por ende, se inscribió en el estudio a los 603 pacientes restantes. Entre estos 603 pacientes, 328 (138 niñas y 190 varones) se sometieron a faringoplastia y amigdalectomía, y conformaron el grupo de estudio, mientras que 275 pacientes (108 niñas y 167 varones) recibieron amigdalectomía solamente, con o sin adenoidectomía, y se los incluyó como el grupo de referencia (Figura 1). Las medias de la edad del grupo de estudio y el grupo de referencia fueron $4,9 \pm 2,1$ años (intervalo: 3-11 años) y 5,13 $\pm 2,4$ años (intervalo: 3-12 años) respectivamente.

Las diferencias entre los dos grupos con respecto a cualquiera de estas variables demográficas no fueron estadísticamente significativas (Tabla 1).

\section{Hemorragia posoperatoria}

En lo que respecta a hemorragia primaria, los resultados obtenidos fueron estadísticamente significativos. La tasa de hemorragia posoperatoria fue del $0,91 \%$ (tres pacientes) en el grupo de estudio y del 4,36\% (12 pacientes) en el grupo de referencia. La comparación de estas proporciones reveló que la diferencia entre estos dos grupos era estadísticamente significativa $(\mathrm{p}=0,008 ;$ Tabla 2). Más aún, en el grupo de referencia, hubo dos casos de hemorragia tardía.

Tabla 1. Datos demográficos

Faringoplastia Amigdalectomía Valor $p$ sola

\begin{tabular}{lccc}
\hline $\begin{array}{l}\text { Cant. de } \\
\text { pacientes }\end{array}$ & 328 & 275 & \\
$\begin{array}{l}\text { Edad } \\
\text { Sexo, }\end{array}$ & $4,9 \pm 2,1(3-11)$ & $5,13 \pm 2,4(3-12)$ & NS \\
$\begin{array}{l}\text { femenino/masculino } \\
\text { IMC } \\
\text { (puntaje Z) }\end{array}$ & $138 / 190$ & $108 / 167$ & NS \\
$\begin{array}{l}\text { Cociente A/N } \\
\text { Tamaño de }\end{array}$ & $0,72 \pm 1,13$ & $0,75 \pm 1,16$ & NS \\
$\begin{array}{l}\text { la amígdala } \\
\text { IAH obstructiva } \\
\text { (/h, todo el }\end{array}$ & $2,22 \pm 0,13$ & $2,25 \pm 0,15$ & NS \\
período de sueño) & $16,2 \pm 4,1$ & $15,95 \pm 4,7$ & NS \\
\hline
\end{tabular}

Cociente A/N: cociente adenoide/nasofaringe;

NS: no significativo. IAH: índice de apnea-hipopnea. 


\section{Características de la herida faríngea}

$\mathrm{Al}$ examinar la garganta cinco días después de la cirugía, se observó una gran diferencia en la cicatrización de la herida faríngea. En los pacientes que se sometieron a faringoplastia y amigdalectomía, las heridas estaban limpias y casi cicatrizadas. Sin embargo, los pacientes del grupo que recibió amigdalectomía, presentaban una cantidad considerable de escaras o pseudomembranas blancas en la fosa amigdalina. Esta afección influyó sobre la hemorragia posamigdalectomía y sobre el tiempo que los pacientes debieron invertir en la fase de recuperación de la operación.

\section{Función velofaríngea posoperatoria}

Respecto de la inteligibilidad del habla posoperatoria, cinco de los pacientes del grupo de estudio y tres del grupo de referencia empeoraron de manera significativa, aunque no se observaron tendencias significativas hacia el empeoramiento cuando se compararon los resultados de ambos grupos $(p=0,733)$. Todos los pacientes se sometieron a una nasofaringoscopia electrónica flexible. No hubo cambios notables en el cierre palatino, el movimiento de la pared lateral y los

TABLA 2. Hemorragia posoperatoria $(\%)$

\begin{tabular}{lccc}
\hline & $\begin{array}{c}\text { Grupo de } \\
\text { estudio } \\
(\mathbf{n}=\mathbf{3 2 8})\end{array}$ & $\begin{array}{c}\text { Grupo de } \\
\text { referencia } \\
(\mathbf{n}=\mathbf{2 7 5 )}\end{array}$ & Valor $\boldsymbol{p}$ \\
\hline Hemorragia primaria & $3(0,91)$ & $10(3,63)$ & 0,025 \\
Hemorragia tardía & $0(0)$ & $2(0,73)$ & 0,208 \\
Todas las hemorragias & $3(0,91)$ & $12(4,36)$ & 0,008 \\
\hline
\end{tabular}

tamaños del espacio velofaríngeo en los datos pre y posamigdalectomía de cada paciente (Tabla 3 y Figura 2). Por lo tanto, la diferencia entre estos dos grupos no fue estadísticamente significativa.

En esta población, no se observaron reflujo nasofaríngeo posoperatorio ni hipernasalidad.

\section{DISCUSIÓN}

Aunque los tratamientos no quirúrgicos se han transformado en una opción cada vez más viable, la cirugía todavía continúa siendo el método de tratamiento más recomendado para niños con apnea obstructiva del sueño. El debate sobre cómo evitar las complicaciones posoperatorias y las molestias asociadas a la cirugía sigue siendo un tema fundamental. Los resultados que obtuvimos respaldaron el rendimiento de la faringoplastia y la amigdalectomía combinadas para el tratamiento de los niños con apnea obstructiva del sueño. Aunque algunos otorrinolaringólogos no aceptan este procedimiento debido a que requiere mayor tiempo de operación y sus ventajas son inciertas, nuestro estudio muestra los beneficios que

TABLA 3. Cambio posoperatorio de la función velofaríngea (\%)

\begin{tabular}{lccc}
\hline & $\begin{array}{c}\text { Grupo de } \\
\text { estudio } \\
(\mathbf{n = 3 2 8})\end{array}$ & $\begin{array}{c}\text { Grupo de } \\
\text { referencia } \\
(\mathbf{n}=\mathbf{2 7 5 )}\end{array}$ & Valor $p$ \\
\hline Empeoramiento de la & & & \\
inteligibilidad del habla & $5(1,52)$ & $3(1,09)$ & 0,733 \\
Insuficiencia velofaríngea & $0(0)$ & $0(0)$ & \\
Reflujo nasofaríngeo & $0(0)$ & $0(0)$ & \\
Hipernasalidad & $4(1,22)$ & $2(0,73)$ & 0,694 \\
\hline
\end{tabular}

Figura 1. Diagrama de flujo del estudio

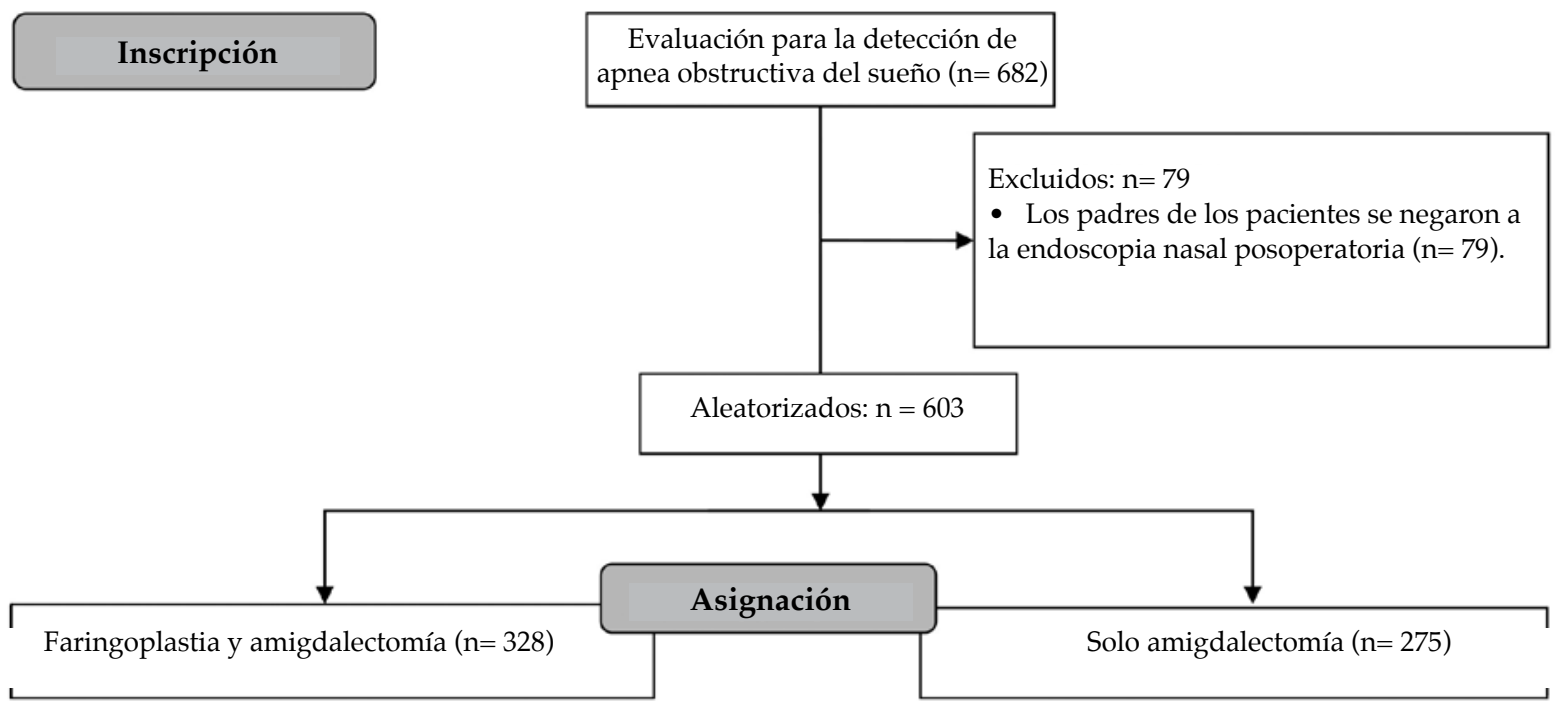


ofrecen la faringoplastia y la amigdalectomía combinadas en la prevención de la hemorragia posoperatoria y la cicatrización de la herida.

La hemorragia posoperatoria es una de las morbilidades principales tras la amigdalectomía, incluidas las hemorragias primaria o reaccionaria (<24 horas) y las infecciones posoperatorias asociadas con hemorragias tardías o secundarias (> 24 horas). ${ }^{16}$

La hemorragia primaria es más violenta y profusa que la secundaria. Además, ocurre cuando los reflejos respiratorios protectores del paciente están atenuados por los efectos de la anestesia o efectos narcóticos. ${ }^{17}$ Los resultados que obtuvimos mostraron que la hemorragia reaccionaria ocurrió con mayor frecuencia que la hemorragia tardía en ambas poblaciones. Más aún, la hemorragia tardía ocurrió principalmente en el grupo de referencia. En el grupo de estudio, las tasas de hemorragia fueron bajas (inferiores al 1\%). Estos resultados quizá estén relacionados con el cierre de la herida amigdalina mediante la sutura de los pilares amigdalinos posterior y anterior, con lo que se evitó la reapertura de vasos sanguíneos dañados en forma intraoperatoria. Además, Lehnerdt, ${ }^{18}$ consideró varios casos de traumatismo nutricional relacionados con hemorragias. El procedimiento que elegimos también puede reducir de manera eficaz la estimulación nutricional posoperatoria.

A partir de la descripción de la primera amigdalectomía que hizo Celsus en 50 a. $C^{19}$ los otorrinolaringólogos se han acostumbrado a no suturar las heridas. En la actualidad, aún no existe la certeza de que este procedimiento sea realmente beneficioso para la cicatrización de la herida. La amigdalectomía produce una herida abierta sujeta a varios factores en un campo altamente contaminado. La cicatrización de la herida depende del crecimiento de la mucosa desde la periferia hacia adentro, proceso que puede continuar durante 1 a 2 semanas después de la cirugía. Durante el proceso, la fosa posoperatoria atraviesa una serie de complejos cambios fisiopatológicos, incluida la formación de coágulos de fibrina, su proliferación y separación del tejido granulado subyacente. ${ }^{20}$

Los pacientes también experimentan diversos niveles de malestar. Todos estos factores pueden retrasar la cicatrización completa. Aunque la mayoría de las heridas abiertas pueden cicatrizarse, es posible acortar el tiempo necesario para la cicatrización y el nivel de malestar. En nuestro estudio, en casi todos los pacientes del grupo de faringoplastia y amigdalectomía, las heridas habían cicatrizado completamente cinco días después de la cirugía, mientras que, en los pacientes del grupo de referencia, la fosa amigdalina continuaba procesando activamente coágulos de fibrina.

Algunos otorrinolaringólogos fueron adoptando la práctica de suturar los pilares amigdalinos después de la amigdalectomía en niños con apnea obstructiva del sueño. Sin embargo, sigue cuestionándose una y otra vez la posibilidad de que este método genere algunos efectos adversos, incluidos efectos negativos, sobre la función velofaríngea posoperatoria.

Habitualmente, la función está determinada por la estructura. Después de las cirugías reconstructivas, incluida la uvulopalatofaringoplastia y la faringoplastia, la modificación estructural en las vías respiratorias altas puede, en cierta medida, afectar la función y la fisiología. Si bien la insuficiencia velofaríngea es una complicación poco frecuente de la uvulopalatofaringoplastia, es un factor de riesgo

FIGURA 2. Vistas nasofaringoscópicas durante la articulación

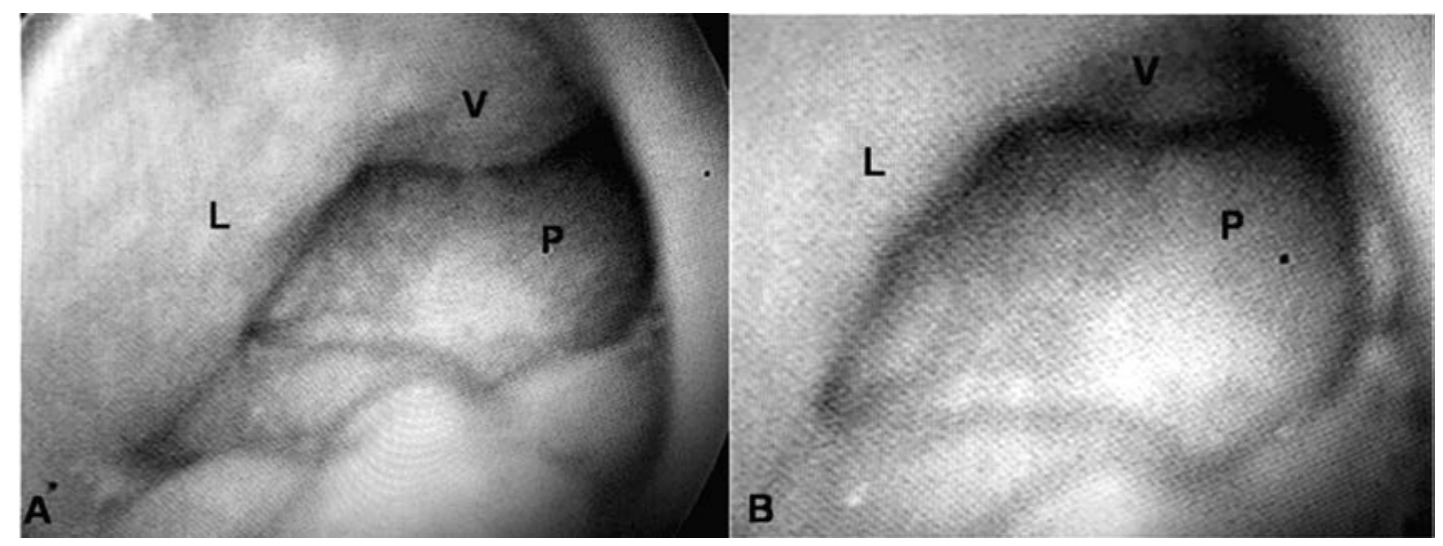

(A) Vista preoperatoria con obliteración del espacio.

(B) Vista posoperatoria con obliteración del espacio (cierre velofaríngeo) L: pared nasofaríngea lateral;

P: pared nasofaríngea posterior; V: velo. 
para los pacientes cuando se hace una resección o cuando los pacientes tienen una movilidad deficiente en el paladar y/o las paredes faríngeas laterales. ${ }^{21}$ Un estudio mostró un riesgo menor de deterioro de la función velofaríngea entre los pacientes que no tenían paladar hendido después de la amigdalectomía con faringoplastia. ${ }^{22}$ En nuestro estudio, intentamos evaluar la función velofaríngea en niños con apnea obstructiva del sueño después de la faringoplastia. No obstante, no se observaron diferencias estadísticas significativas en comparación con el grupo de referencia. Por lo tanto, este procedimiento no implica un mayor riesgo de insuficiencia velofaríngea.

La controversia sobre la fisiopatología exacta de la apnea obstructiva del sueño en los niños continúa, pero la mayoría de los especialistas parecen aceptar la teoría de que la permeabilidad de las vías respiratorias superiores se ve limitada o se reduce notablemente durante la inspiración. Dicha reducción es el resultado integral de un conjunto de interacciones multifactoriales, que incluye factores funcionales y anatómicos. Según esta teoría, es muy importante tratar de ampliar el estrechamiento al máximo y disminuir la capacidad de reducción del área faríngea tensionando la vía respiratoria flácida en vez de extirpar solo los tejidos adenoides y amigdalinos. Algunos autores ${ }^{23}$ defienden la práctica de suturar la herida amigdalina para agrandar al máximo el espacio aéreo retropalatino y, así, disminuir la capacidad de reducción de la pared faríngea lateral y el paladar blando. El efecto a largo plazo de suturar la herida amigdalina para aliviar la apnea obstructiva del sueño no se ha investigado.

\section{CONCLUSIÓN}

En nuestro estudio, la amigdalectomía combinada con faringoplastia ofrecieron ventajas significativas en el período posoperatorio en los pacientes pediátricos con apnea obstructiva del sueño. En comparación con la amigdalectomía sola, el tratamiento combinado reduce el tiempo de cicatrización de la herida y la proporción de hemorragias posoperatorias. Además, el riesgo de insuficiencia velofaríngea no aumentó con este método. En nuestra opinión, estos resultados indican que la combinación de faringoplastia y amigdalectomía conlleva un gran potencial para el tratamiento de la apnea obstructiva del sueño en pacientes pediátricos. Además, la ventaja que implica un ahorro potencial en los costos merece continuar las investigaciones.

\section{REFERENCIAS}

1. Lumeng JC, Chervin RD. Epidemiology of pediatric obstructive sleep apnea. Proc Am Thorac Soc. 2008; 5(2):242-52.

2. Schwengel DA, Dalesio NM, Stierer TL. Pediatric obstructive sleep apnea. Anesthesiol Clin 2014;32(1):237-61.
3. Arali V, Namineni S, Sampath Ch. Pediatric obstructive sleep apnea syndrome: time to wake up. Int J Clin Pediatr Dent 2012; 5(1):54-60.

4. Zhu J, Fang Y, Chen X, et al. The impacts of obstructive sleep apnea hypopnea syndrome severity and surgery intervention on psychological and behavioral abnormalities and postoperative recovery in pediatric patients. Med Sci Monit 2014; 20:1474-80.

5. Slaats MA, Van Hoorenbeeck K, Van Eyck A, et al. Upper airway imaging in pediatric obstructive sleep apnea syndrome. Sleep Med Rev 2015; 21:59-71.

6. Mukhatiyar P, NandalikeK, Cohen HW, et al. Intracapsular and Extracapsular Tonsillectomy and Adenoidectomy in Pediatric Obstructive Sleep Apnea. JAMA Otolaryngol Head Neck Surg 2016; 142(1):25-31.

7. Reusser NM, Bender RW, Agrawal NA, et al. Posttonsillectomy hemorrhage rates in children compared by surgical technique. Ear Nose Throat J 2017; 96(7):e7-11.

8. Ferary M, Biet A, Strunski V, et al. Impact of the mode of hospitalisation on the postoperative complication rate after dissection tonsillectomy in children. Eur Ann Otorhinolaryngol Head Neck Dis 2014; 131(6):345-9.

9. Friedman M, Wilson MN, Friedman J, et al. Intracapsular coblation tonsillectomy and adenoidectomy for the treatment of pediatric obstructive sleep apnea/hypopnea syndrome. Otolaryngol Head Neck Surg 2009; 140(3):358-62.

10. Kirazli T, Bilgen C, Midilli R, et al. Bipolar electrodissection tonsillectomy in children. Eur Arch Otorhinolaryngol 2005; 262(9):716-8.

11. D'Eredità R, Marsh RR. Contact diode laser tonsillectomy in children. Otolaryngol Head Neck Surg 2004; 131(5):732-5.

12. Shen $\mathrm{L}$, Zheng B, Lin Z, et al. Tailoring therapy to improve the treatment of children with obstructive sleep apnea according to grade of adenotonsillar hypertrophy. Int $J$ Pediatr Otorhinolaryngol 2015; 79(4):493-8.

13. Acar M, Kankilic ES, Koksal AO, et al. Method of the diagnosis of adenoid hypertrophy for physicians: adenoidnasopharynx ratio. J Craniofac Surg 2014; 25(5):e438-40.

14. Paulson LM, MacArthur CJ, Beaulieu KB, et al. Speech Outcomes after Tonsillectomy in Patients with Known Velopharyngeal Insufficiency. Int J Otolaryngol 2012; 2012:912767.

15. Tieu DD, Gerber ME, Milczuk HA, et al. Generation of consensus in the application of a rating scale to nasendoscopic assessment of velopharyngeal function. Arch Otolaryngol Head Neck Surg 2012; 138(10):923-8.

16. Amoils M, Chang KW, Saynina O, et al. Postoperative Complications in Pediatric Tonsillectomy and Adenoidectomy in Ambulatory vs Inpatient Settings. JAMA Otolaryngol Head Neck Surg 2016; 142(4):344-50.

17. Lou ZC, Lou ZH. Post-tonsillectomy hemorrhage: Underlying factors and prevention. Am J Otolaryngol 2018; 39(2):230-1.

18. Lehnerdt G, Senska K, Jahnke K, et al. Post-tonsillectomy haemorrhage: a retrospective comparison of abscess- and elective tonsillectomy. Acta Otolaryngol 2005; 125(12):1312-7.

19. Mixon CM, Weinberger PM, Austin MB. Comparison of microdebrider subcapsular tonsillectomy to harmonic scalpel and eletrocautery total tonsillectomy. Am J Otolaryngol 2007; 28(1):13-7.

20. Isaacson G. Tonsillectomy Care for the Pediatrician. Pediatrics 2012; 130(2):324-34.

21. Abdel-Aziz M. The effectiveness of tonsillectomy and partial adenoidectomy on obstructive sleep apnea in cleft palate patients. Laryngoscope 2012; 122(11):2563-7.

22. Mora R, Crippa B, Dellepiane M, et al. Effects of adenotonsillectomy on speech spectrum in children. Int J Pediatr Otorhinolaryngol 2007; 71(8):1299-304.

23. Lim DJ, Kang SH, Kim BH, et al. Treatment of obstructive sleep apnea syndrome using radiofrequencyassisted uvulopalatoplasty with tonsillectomy. Eur Arch Otorhinolaryngol 2013; 270(2):585-93. 